\title{
Side busking of the cantilever beam with narrow rectangular cross section
}

\author{
Serdar Yazyev, Ivan Zotov, Dmitriy Vysokovsky and Batyr Yazyev* \\ Don State Technical University, 162 Sotcialisticheskaya str., 344022, Russia
}

\begin{abstract}
The problem of lateral buckling of a cantilever strip with a constant narrow cross section loaded with a concentrated force at the end of the span is considered. In the study of lateral buckling of beam energy method was used. For the case of load application in the center of gravity, the problem is reduced to a generalized secular equation. The relationship between the magnitude of the critical force and the position of the point of application of the load. A comparison of the results obtained by the authors with an analytical solution using infinite series and a numerical iterative method is shown.
\end{abstract}

\section{Introduction}

As it is known, many studies are devoted to issues of sustainability, and they cannot be neglected when calculating and designing. In modern calculations of bridges, high-rise buildings, in mechanical engineering and aircraft industry, we are faced with a variety of problems of sustainability, for which it is possible, both local buckling and bulging in general [1-4].

This article considers the case when the console is bent by the force $\mathrm{F}$ applied in the center of gravity of the end section in the plane of the wall. Considering the load, we finally reach the condition when the shape of the bend in the plane of the wall becomes unstable and a bulging occurs, as shown in fig. 1.

To determine the critical value of the load, we assume that a small lateral buckling has occurred, and we determine from the equilibrium equations of the bulging cantilever the smallest load that keeps it in a slightly curved form. This will be a critical load.

\section{Methods}

Consider the system of fixed axes of coordinates $X, Y, Z$ on the left end of the beam and the system of axes $v, u$ in an arbitrary section $m-n$ of the console.

\footnotetext{
* Corresponding author: ibm2225101@gmai.com
} 


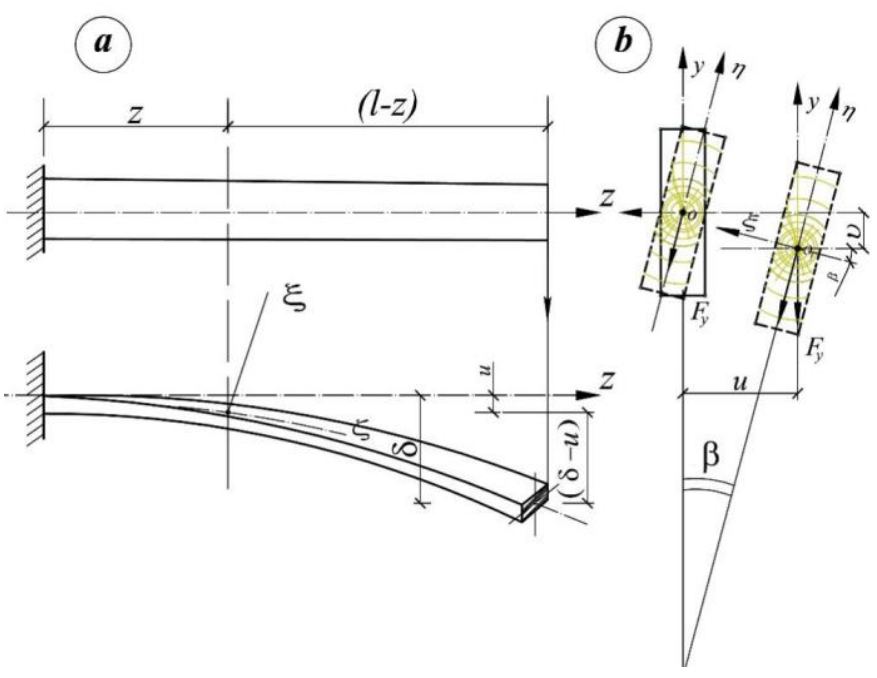

Fig. 1. - Design diagram of a flat bend

The deformation of the strip is determined by two components of the displacement $-u, v$ as well as the angle $\beta$, by which it rotates $[5,6]$. Considering the part of the console to the right of the cross section $\mathrm{m}-\mathrm{n}$ and determining the moments from the vertical load $\mathrm{F}$ relative to the axes parallel to $X, Y, Z$ passing through the center of gravity of the cross section, we get:

$$
M_{x}=F(l-x) ; \quad M_{y}=0 ; M_{z}=-F(\delta-u) ;
$$

Projecting these moments on the axis $\xi, \eta, \zeta$ using the cosine table [4], we represent the moments in new coordinates:

$$
\left\{\begin{array}{c}
M_{\xi}=F(l-z)+F(\delta-u) \frac{d u}{d z} \\
M_{\eta}=-F(l-z) \beta+F(\delta-u) \frac{d v}{d z} \\
M_{\xi}=F(l-z) \frac{d u}{d z}-F(\delta-u)
\end{array}\right.
$$

Neglecting small values of higher order, we get:

$$
\left\{\begin{array}{c}
M_{\xi}=F(l-z) \\
M_{\eta}=-F(l-z) \beta \\
M_{\xi}=F(l-z) \frac{d u}{d z}-F(\delta-u)
\end{array}\right.
$$

Differentiating the third equation (2) by $z$ and excluding using the first equation $\frac{d^{2} u}{d z^{2}}$, we obtain the following equation for determining the relative angle $\beta$ : 


$$
\frac{d^{2} \beta}{d z^{2}}+\frac{F^{2}}{B_{1} C}(l-z)^{2} \beta=0
$$

where $B_{1}$ - flexural rigidity of the plane $X Z, C$ - torsional stiffness, $\beta$-torsion angle.

\section{Solution methods}

A numerical solution in a finite difference scheme and a solution in infinite series gives the result of the critical force [4]:

$$
F_{c r}=\frac{4,013}{l^{2}} \sqrt{B_{1} C}
$$

Note that formula (4) gives the correct load value only in the elastic range. Beyond the limit of elasticity, buckling occurs under load, less than that given by the formula (4).

In the study of flat bending, you can apply energy method with engineering precision. Consider the deformation of the beam shown in (Fig. $1 \mathrm{~b}$ ).

Under the action of a load close to critical, the transition from the plane of buckling to lateral buckling entails fractal transitions, that is, the transfer of the energy of an external load to the potential energy of deformation. When the beam bulges to the side, the deformation energy of the beam increases. This is facilitated by the bending of the beam in the plane of action of the concentrated force in the transverse direction and torsion around the longitudinal axis $z$. At the same time, the load point decreases, and the load does some work:

$$
U_{\text {bend }}+U_{\text {torsion }}=U_{F}
$$

The critical load value is now determined from the condition that this work is equal to the sum of the energies - the lateral bending deformation energy and the torsional deformation energy.

The potential energy of lateral bending (relatively $\eta$ ) equal to:

$$
\begin{gathered}
U_{\text {bend }}=\int_{0}^{l} \frac{M_{y}^{2}(z) d z}{2 B_{1}} \\
M_{y}=F(l-x) \sin \beta \approx F(l-x) \beta
\end{gathered}
$$

Thus, the energy of lateral bending looks like:

$$
U_{\text {bend }}=\frac{F^{2}}{2 B_{1}} \int_{0}^{l}(l-x)^{2} \beta^{2} d z
$$

When torsion potential energy is expressed through the relative angle of twist $\beta$. Work torque on an infinitely small length $d z$ has the form:

$$
d U_{\text {torsion }}=\frac{M_{\zeta} d \beta}{2}, \quad d \beta=\frac{M_{\zeta} d \beta}{C} \rightarrow M_{\zeta}=C \frac{d \beta}{d z} \quad d \beta=\frac{d \beta}{d z} d z
$$

Then finally for the potential energy of torsion, accumulated during lateral buckling of the cantilever beam, is equal to: 


$$
\begin{gathered}
d U_{\text {torsion }}=\frac{C}{2} \frac{d \beta}{d z} \frac{d \beta}{d z} d z=\frac{C}{2}\left(\frac{d \beta}{d z}\right)^{2} d z \\
U_{\text {torsion }}=\frac{C}{2} \int_{0}^{l}\left(\frac{d \beta}{d z}\right)^{2} d z
\end{gathered}
$$

To determine the reduction of the load $F$ during lateral buckling, consider the cross section of the element before and after buckling (Fig. 1, b). We will show, in this case, that the movement of a point $O$ to a position $O_{l}$ is caused by a combination of two factors, in particular, lateral displacement in the direction of the axis $O \xi$ and rotation of the section around an angle $\beta$. The reason for moving the point (lowering the point) of application of force is the flat form of the bend $(Z O Y)$.

According to the well-known Castigliano theorem, the vertical displacement of a point $O$ is determined from the minimization of the relation (5) in terms of external load:

$$
v=\frac{d U_{b e n d}}{d F}=\frac{F}{E I_{y}} \int_{0}^{l}(l-x)^{2} \beta^{2} d z ;
$$

Then the work of the external load (which reached the value $F_{c r}$ at the moment of the lateral displacement that began) on the movement $v$ will be:

$$
U_{F}=F v=\frac{F^{2}}{B_{1}} \int_{0}^{l}(l-x)^{2} \beta^{2} d z
$$

The equation for determining the critical load becomes as follows:

$$
\begin{gathered}
\frac{F^{2}}{2 B_{1}} \int_{0}^{l}(l-x)^{2} \beta^{2} d z+\frac{C}{2} \int_{0}^{l}\left(\frac{d \beta}{d z}\right)^{2} d z=\frac{F^{2}}{B_{1}} \int_{0}^{l}(l-x)^{2} \beta^{2} d z \\
\frac{C}{2} \int_{0}^{l}\left(\frac{d \beta}{d z}\right)^{2} d z=\frac{F^{2}}{2 B_{1}} \int_{0}^{l}(l-x)^{2} \beta^{2} d z \\
F_{c r}=\left(\frac{C B_{1}}{2} \int_{0}^{l}\left(\frac{d \beta}{d z}\right)^{2} d z\right) /\left(\int_{0}^{l}(l-x)^{2} \beta^{2} d z ;\right)
\end{gathered}
$$

To determine the critical value of the load $F_{c r}$, it is necessary to take a suitable analytical expression for the torsion angle that satisfies the conditions at the ends of the beam, and substitute it into equation (9). 


\section{Results and Discussion}

The general expression for $\beta$, satisfying the conditions at the ends can be taken in the form of a trigonometric series $[8,9]$ :

$$
\beta=a_{1}\left(1-\cos \left(\frac{\pi z}{l}\right)\right)
$$

Using for $\beta$ as first approximation only the first term of this series, substituting it into equation (9)

$$
\begin{gathered}
\frac{d \beta}{d z}=a_{1} \frac{\pi}{l} \sin \left(\frac{\pi z}{l}\right) \\
F_{c r}=\left(\frac{C B_{1}}{2} \int_{0}^{l} a_{1}^{2}\left(\frac{\pi}{l}\right)^{2}\left(\sin \left(\frac{\pi z}{l}\right)\right)^{2} d z\right) /\left(\int_{0}^{l}(l-x)^{2}\left(a_{1} \frac{\pi}{l} \sin \left(\frac{\pi z}{l}\right)\right)^{2} d z\right)
\end{gathered}
$$

and integrating, we get:

$$
F_{c r}=\frac{\sqrt{2} \pi^{2} \sqrt{\frac{B_{1} C}{l^{4}}}}{\sqrt{\pi^{2}+2}}=4,05 \sqrt{\frac{B_{1} C}{l^{4}}}
$$

The numerical solution in the finite-difference scheme and the solution using the energy method gives a difference in the results of the critical force of $0.91 \%$.

\section{Summary}

Thus, the last example shows that the energy method can be successfully applied in the study of lateral buckling of beams. We add that when using the energy method, the complex integration of differential equations by the method of infinite series is replaced by the calculation of simple integrals, included in equation (9), and a relatively simple expression for $\beta$ usually gives $F_{c r}$ with an accuracy sufficient for practical purposes. Usually $F_{c r}$, determined by the energy method, is always greater than its true value.

\section{References}

1. B.M. Yazyev, A. S. Chepurnenko et al., Materials Science Forum, 935 (2018) 144-149.

2. A.V. Ishchenko, I.M. Zotov Inženernyj vestnik Dona, 1 (2019)

3. A. S. Volmir, Stability of deformable systems (Nauka, 1967)

4. S. P. Timoshenko, J. M. Gere, Theory of elastic stability (McGraw-Hill, 1961)

5. A. A. Lukin, I. S. Kholopov, Procedia Engineering, 153 (2016) 414-418.

6. A.O. Lukin, A.A. Suvorov, Construction of Unique Buildings and Structures, 2 (2016) 45-67.

7. I. M. Balzannikov, I.S. Kholopov et al., Procedia Engineering. 111 (2015) 74-81.

8. A.S. Chepurnenko, V.V. Ulianskaya, D.A. Vysokovsky, I.M. Zotov, Inženernyj vestnik Dona, 2 (2018)

9. A. S. Chepurnenko, V.V. Ulianskaya, S.B. Yazyev, I.M. Zotov, MATEC, 196 (2018) 01003 